\title{
Terahertz Generation in an Electrically Biased Optical Fiber: A Theoretical Investigation
}

\author{
Montasir Qasymeh \\ Department of Electrical and Computer Engineering, Abu Dhabi University, Abu Dhabi, UAE \\ Correspondence should be addressed to Montasir Qasymeh, montasir.qasymeh@adu.ac.ae \\ Received 28 May 2012; Accepted 13 August 2012 \\ Academic Editor: Roberto Morandotti
}

Copyright () 2012 Montasir Qasymeh. This is an open access article distributed under the Creative Commons Attribution License, which permits unrestricted use, distribution, and reproduction in any medium, provided the original work is properly cited.

We propose and theoretically investigate a novel approach for generating terahertz $(\mathrm{THz})$ radiation in a standard single-mode fiber. The optical fiber is mediated by an electrostatic field, which induces an effective second-order nonlinear susceptibility via the Kerr effect. The THz generation is based on difference frequency generation (DFG). A dispersive fiber Bragg grating (FBG) is utilized to phase match the two interacting optical carriers. A ring resonator is utilized to boost the optical intensities in the biased optical fiber. A mathematical model is developed which is supported by a numerical analysis and simulations. It is shown that a wide spectrum of a tunable $\mathrm{THz}$ radiation can be generated, providing a proper design of the FBG and the optical carriers.

\section{Introduction}

Due to a lack of generation and detection instrumentation, the electromagnetic spectrum between infrared light and microwave radiation, traditionally known as the terahertz $(\mathrm{THz})$ gap, has not been fully explored [1]. The application of THz radiation was traditionally limited to astronomy and analytical science.

Recent advances in photonics have laid the groundwork for the realization of $\mathrm{THz}$ sources and detectors for applications in biomedical imaging [2] and ultra-fast communications [3]. As $\mathrm{THz}$ sources become more readily available, $\mathrm{THz}$ technology is being increasingly used in a variety of fields, including information and communications technology, biology and medical sciences, nondestructive evaluation, homeland security, quality control of food and agriculture, global environmental monitoring, and ultrafast computing, to mention a few examples [4]. The wide and crucial applications of $\mathrm{THz}$ waves are due to its unique way of interacting with materials. For example, in medical science, the ability of $\mathrm{THz}$ wave to probe intermolecular interactions enables it to provide both structural and functional information. Consequently, and considering its safe, accurate, and economical features, $\mathrm{THz}$ radiation promises to alternate other scanning methods such as high frequency ultrasound, magnetic resonance imaging, and near-infrared imaging [4].
This promising technology has the potential to lead the way many diseases are diagnosed and ultimately cured.

In the past few years, several techniques have been proposed to generate $\mathrm{THz}$ waves. Generation of $\mathrm{CW}$ and pulsed $\mathrm{THz}$ waves have been both investigated. Techniques to generate $\mathrm{CW} \mathrm{THz}$ waves include quantum cascade laser (QCL) [5], directly multiplied source [6], backward wave oscillator (BWO) [7], germanium laser [8], and silicon impurity state laser [9]. Similarly, techniques to generate pulsed $\mathrm{THz}$ waves include nonlinear optical source (which can also be utilized to generate $\mathrm{CW} \mathrm{THz}$ waves) [10], optically pumped $\mathrm{THz}$ laser [11], and free electron laser [12]. THz generation based on a QCL is realized based on intersubband transitions in quantum wells. Although a QCL can provide strong $\mathrm{THz}$ waves, its tunability is limited [5]. Generating $\mathrm{THz}$ wave utilizing a direct multiplied source is achieved by the mean of submillimetre wave multiplication [6]. The multiplication can be done in a biased GaAs crystal. Mainly, this approach is limited to generating $\mathrm{THz}$ wave at a low frequency. A BWO source has less impetus from a practical point of view, since it has a stringent requirement for the power supply. In addition, a BWO also has a vacuum tube which is fragile. $\mathrm{THz}$ generation based on optically pumped $\mathrm{THz}$ laser, on the other hand, has a small efficiency (less than $0.1 \%$ ) resulting in a significant heat loading. A germanium laser can generate a $\mathrm{THz}$ wave with narrow 
linewidth, but it requires liquid helium and a pulsed magnet system making the system costly. A silicon impurity state laser can generate a $\mathrm{THz}$ wave with a power up to tens of $\mathrm{mW}$, but it is usually operating at a temperature below $20 \mathrm{~K}$, and the operation is limited to a pulsed mode. A free electron laser can generate a $\mathrm{THz}$ wave with a large tunable range, but it has issues such as large size, high cost, and great complexity. On the other hand, the main nonlinear optical methods for generating $\mathrm{THz}$ radiation [4] include optical rectification [13], parametric conversion [14], and laser generated plasma filament [15]. The optical rectification technique can be used to generate a $\mathrm{THz}$ wave in a frequency range from 0.3 to $30 \mathrm{THz}$, and it is a common device for broadband $\mathrm{THz}$ generation at room temperature. The parametric conversion technique is based on the mixing of laser beams to generate a beat frequency that is in the $\mathrm{THz}$ range. The frequency of the $\mathrm{THz}$ wave is tunable and can operate in the $\mathrm{CW}$ regime with narrow linewidth. Generating $\mathrm{THz}$ waves using lasergenerated plasma filament technique is achieved by the mean of four wave mixing (FWM) process. An intensive laser input is needed though.

Developing a high-power, low-cost, fast tunable, and high reliable $\mathrm{THz}$ source is one of most challenging issues in a $\mathrm{THz}$ system. For example, for medical applications, a $\mathrm{THz}$ source is required to have a frequency between 0.3 and $3 \mathrm{THz}$ in addition to all other features mentioned above. Among the $\mathrm{THz}$ sources currently available, the parametric conversion technique could provide a $\mathrm{THz}$ source with a narrow line-width and can operate at room temperature. It is also fast to tune at a relatively low cost [16]. However, the phase-mismatching dilemma is an issue that would decrease the generation efficiency. One common way to increase the efficiency is to boost the pumping power by the use of a pulsed source. Unfortunately, this approach is limited by the two-photon absorption [17]. On the other hand, utilizing a periodic structure is an effective way to achieve quasiphase matching and thus improves the conversion efficiency. Usually, noncentrosymmetric crystals in free space geometry are employed [13-17]. Despite the achievable high generated power, this scheme lacks the advantages of compatibility with fiber and integrated optics and is sensitive to environmental effects.

In this paper, we propose a novel approach to generating a $\mathrm{THz}$ wave based on parametric conversion utilizing an optical fiber. Indeed, $\mathrm{THz}$ generation in an optical fiber has recently been reported [18]. There, the $\mathrm{THz}$ generation is achieved based on the photo-Dember effect. However, the achieved tunability is limited. In our proposed work, the optical fiber is electrostatically biased to induce an effective second-order nonlinear susceptibility via the Kerr effect [19]. Thus, a wide frequency-tunable range can be achieved given the off-resonance nature of the Kerr effect. A fiber Bragg grating (FBG) is used to phase match the two interacting light waves, thanks to the dispersion properties of the FBG. On the other hand, given the weak nonlinearity of the optical fiber, the generation efficiency is expected relatively low. The efficiency is improved by increasing the biasing electrostatic field. The maximum electrostatic field can be very high and is only limited by the electric field strength of the optical fiber material (i.e., $30 \mathrm{kV} / \mathrm{mm}$ for fused silica optical fibers). In addition, the two-photon absorption is negligible in the optical fiber, and thus an intensive optical input can also be used to increase the generation efficiency. In the scheme presented in this paper, a ring resonator, incorporating a polarization beam coupler, is utilized to boost the optical intensities. We note here that, for a practical system, a poled fiber with internal electrostatic field (that can be as strong as the electric field strength of the material) can be used instead of having an external electrostatic field bias [20]. The proposed scheme using an optical fiber for $\mathrm{THz}$ generation has the advantages of compatibility with other fiber optic devices, simplicity, low cost, and high potential for integration. For instant, the proposed system can find application in in-vivo $\mathrm{THz}$ scanning.

The remainder of the paper is arranged as follows. In Section 2, a theoretical model is presented, in which the generation of $\mathrm{THz}$ radiation in an electrostatically biased optical fiber is modeled. In Section 3, a numerical simulation is presented. Realistic parameters are selected and are used in the simulation. Finally, a conclusion is drawn in Section 4.

\section{Theoretical Modeling}

Consider two optical carriers are propagating in an optical fiber, and the optical fiber is mediated by an electrostatic field $E_{\mathrm{dc}}$. The electric field inside the optical fiber can thus be written as

$$
E(t)=A_{1} e^{j\left(k_{1} z-\omega_{1} t\right)}+A_{2} e^{j\left(k_{2} z-\omega_{2} t\right)}+E_{\mathrm{dc}}+\text { c.c. },
$$

where $A_{1}, A_{2}$ and $\omega_{1}, \omega_{2}$ are the amplitudes and frequencies of the two optical carriers. Here the electrostatic field is considered collinear with the polarization of the propagating light modes.

The nonlinear polarization field, generated by the Kerr type nonlinear medium, such as an optical fiber, can be written as [21]

$$
P_{\mathrm{NL}}=\varepsilon_{0} \chi^{(3)} \vdots \mathrm{EEE}
$$

where $\varepsilon_{0}$ is the free space permittivity and $\chi^{(3)}$ is the thirdorder dielectric susceptibility.

Substituting (1) into (2), we have the nonlinear polarization at frequencies $\omega_{1}, \omega_{2}$, and $\omega_{3}=\omega_{1}-\omega_{2}$, which is expressed as

$$
P_{\mathrm{NL}}=P_{1} e^{j\left(k_{1} z-\omega_{1} t\right)}+P_{2} e^{j\left(k_{2} z-\omega_{2} t\right)}+P_{3} e^{j\left(k_{3} z-\omega_{3} t\right)} .
$$

Here

$$
\begin{gathered}
P_{1}=\varepsilon_{0} \chi^{(3)}\left[\alpha A_{3} A_{2} E_{\mathrm{dc}} e^{-j\left(k_{1}-k_{2}+k_{3}\right) z}+E_{\mathrm{dc}}^{2} A_{1}\right. \\
\left.+\left|A_{1}\right|^{2} A_{1}+2\left(\alpha^{2}\left|A_{3}\right|^{2}+\left|A_{2}\right|^{2}\right) A_{1}\right], \\
P_{2}=\varepsilon_{0} \chi^{(3)}\left[\alpha A_{1} A_{3}^{*} E_{\mathrm{dc}} e^{j\left(k_{1}-k_{2}-k_{3}\right) z}+E_{\mathrm{dc}}^{2} A_{2}\right. \\
\left.+\left|A_{2}\right|^{2} A_{2}+2\left(\alpha^{2}\left|A_{3}\right|^{2}+\left|A_{1}\right|^{2}\right) A_{2}\right], \\
P_{3}=\varepsilon_{0} \chi^{(3)}\left[5 A_{1} A_{2}^{*} E_{\mathrm{dc}} e^{j\left(k_{1}-k_{2}-k_{3}\right) z}\right],
\end{gathered}
$$


where $k_{i}=\omega_{i} n_{i} / c, i \in(1,2,3)$, and $\alpha$ is the ratio of the $\omega_{3}$-mode inside the fiber (i.e., the ratio of the fiber to the unguided $\omega_{3}$-mode cross sections). Note in (3) that all other polarization terms (i.e., the second harmonic, the third harmonic, and the sum frequency terms) suffer from the phase mismatching and can thus be neglected. However, a proper technique will be utilized to phase match the difference frequency polarization term $P_{3}$. The nonlinear polarization $P_{i}$ can be considered a source of new fields at the $\omega_{i}$ frequency. It then follows that a $\mathrm{THz}$ waves at $\omega_{3}$ can be generated, given that the frequency spacing between the optical carriers $\omega_{1}$ and $\omega_{2}$ is in the THz range. However, the generated $\mathrm{THz}$ wave cannot be guided by the optical fiber and will diffract into free space.

We first model the generation of the $\mathrm{THz}$ wave at $\omega_{3}$, and then the space diffraction effect will be taken into account. The nonlinear wave equation, governing beam propagation in an optical fiber, can be cast into the form:

$$
\nabla^{2} E-\frac{1}{\varepsilon_{0} c^{2}} \frac{\partial^{2} D}{\partial t^{2}}=\mu_{0} \frac{\partial^{2} P_{\mathrm{NL}}}{\partial t^{2}},
$$

where $D$ is the electric displacement, $\mu_{0}$ is the free space permeability, and $c$ is the speed of light in vacuum. Substituting (1) and (3) into (5), we have the slow-varying amplitudes for the three waves, given by

$$
\begin{gathered}
\frac{\partial A_{1}}{\partial z}=\frac{j \chi^{(3)} \omega_{1}}{2 c n_{1}}\left[\alpha A_{3} A_{2} E_{\mathrm{dc}} e^{-j\left(k_{1}-k_{2}-k_{3}\right) z}+E_{\mathrm{dc}}^{2} A_{1}\right. \\
\left.+\left|A_{1}\right|^{2} A_{1}+2\left(\alpha^{2}\left|A_{3}\right|^{2}+\left|A_{2}\right|^{2}\right) A_{1}\right], \\
\frac{\partial A_{2}}{\partial z}=\frac{j \chi^{(3)} \omega_{2}}{2 c n_{2}}\left[\alpha A_{1} A_{3}^{*} E_{\mathrm{dc}} e^{j\left(k_{1}-k_{2}-k_{3}\right) z}+E_{\mathrm{dc}}^{2} A_{2}\right. \\
\left.+\left|A_{2}\right|^{2} A_{2}+2\left(\alpha^{2}\left|A_{3}\right|^{2}+\left|A_{1}\right|^{2}\right) A_{2}\right], \\
\frac{\partial A_{3}}{\partial z}=\frac{j \chi^{(3)} \omega_{3}}{2 c n_{3}} 5 A_{1} A_{2}^{*} E_{\mathrm{dc}} e^{i\left(k_{1}-k_{2}-k_{3}\right) z},
\end{gathered}
$$

where $A_{3}$ is the amplitude of the generated $\mathrm{THz}$ wave.

As can be seen in (6), the phase mismatching dilemma would limit the $\mathrm{THz}$ wave generation. The phase matching condition is given by

$$
k_{1}-k_{2}=k_{3}
$$

We thus propose to utilize a FBG to ensure the phase matching for the $\mathrm{THz}$ generation. As shown in Figure 1, the frequencies of the two optical carriers at $\omega_{1}$ and $\omega_{2}$ lie outside the reflection band of the FBG, but one of the optical carriers, say $\omega_{1}$, is close enough to the reflection band and is, thus, affected by the dispersion of the FBG. It then follows that the effective propagation constant of the optical carrier at $\omega_{1}$ is given by $[22,23]$

$$
k_{1}=k_{B}+\sqrt{\delta_{1}^{2}-\kappa^{2}},
$$

where $k_{B}=\omega_{\mathrm{FBG}} n_{\mathrm{eff}} / c$ is the propagation constant of the FBG, $\omega_{\mathrm{FBG}}, n_{\mathrm{eff}}$, and $\kappa$ are the central frequency, the effective

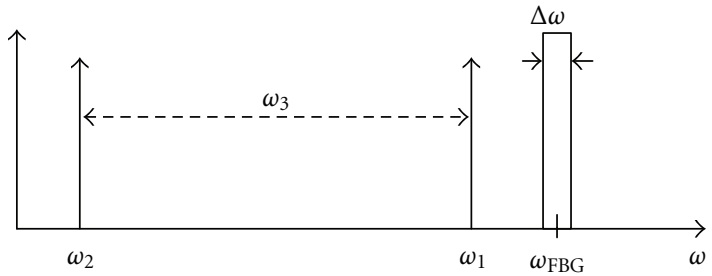

FIGURE 1: The relationship between the reflection spectrum of the FBG and the two optical carriers.

refractive index, and the coupling coefficient of the FBG, respectively. Here $\delta_{1}=\left(n_{1} / c\right)\left(\omega_{1}-\omega_{\mathrm{FBG}}\right)$. Thus, by a proper design of the FBG and the locations of the two optical carriers, the phase matching condition can be satisfied.

Let us assume now that the $\mathrm{THz}$ radiation is generated in the fiber, and consequently the $\mathrm{THz}$ radiation diffracts into the free space. To model the $\mathrm{THz}$ radiation propagation, the generation fiber is divided into small segments, each segment with a length of $\Delta L$. It then follows that the diffracted $\mathrm{THz}$ radiation, which is generated by one $\Delta L$ segment, can be described using the Gaussian beam model [24],

$$
W(z)=W_{0}\left[1+\left(\frac{z}{z_{0}}\right)^{2}\right]^{1 / 2}
$$

where $W(z)$ is the beam width after propagating a distance $z, W_{0}=\sqrt{A_{\text {eff }} / 2 \pi}$ and $z_{0}=W_{0}^{2} \omega_{3} /(2 c)$. Here, $A_{\text {eff }}$ is the effective mode area of the single-mode fiber.

The power of the $\mathrm{THz}$ radiation generated by a fiber with a length of $L=P \times \Delta L$, collected utilizing a lens of a radius of $\mathfrak{R}$, is given by

$$
I_{\text {coll }}=\sum_{p=1}^{P} \frac{\mathfrak{R}}{W\left(z_{p}\right)} I_{p},
$$

where $I_{\text {coll }}$ is the collected power, $z_{p}$ is the distance between the $p$ th segment and the lens, and $I_{p}$ is the power generated by the $p$ th segment.

\section{Numerical Simulation}

A numerical simulation is performed to evaluate the $\mathrm{THz}$ generation. Realistic optical fiber parameters are employed for the simulation. Specifically, we consider $\chi^{(3)}=2.4 \times$ $10^{-22} \mathrm{~m}^{2} / \mathrm{V}^{2}, A_{\text {eff }}=20 \mu \mathrm{m}^{2}$ [19], and a $3 \mathrm{~cm}$ biased optical fiber. We also assume that the FBG center frequency is $f_{\mathrm{FBG}}=193.36 \mathrm{THz}$, the bandwidth is $\Delta f=25 \mathrm{GHz}$, and the coupling coefficient is $\kappa=400 \mathrm{~m}^{-1}$.

Following the expression in (8) and the condition in (7), a $4 \mathrm{THz}$ wave can be generated in the biased optical fiber by mixing two optical carriers of the frequencies of $f_{1}=$ $192.8 \mathrm{THz}$ and $f_{2}=188.8 \mathrm{THz}$.

The power of the collected $\mathrm{THz}$ radiation is simulated, with the results shown in Figure 2. Here, we solve numerically the wave equations given by (6) considering a $3 \mathrm{~cm}$ optical fiber. Then, we calculate the power generated by every $1 \mathrm{~mm}$ length and used (9) and (10) to calculate the power 


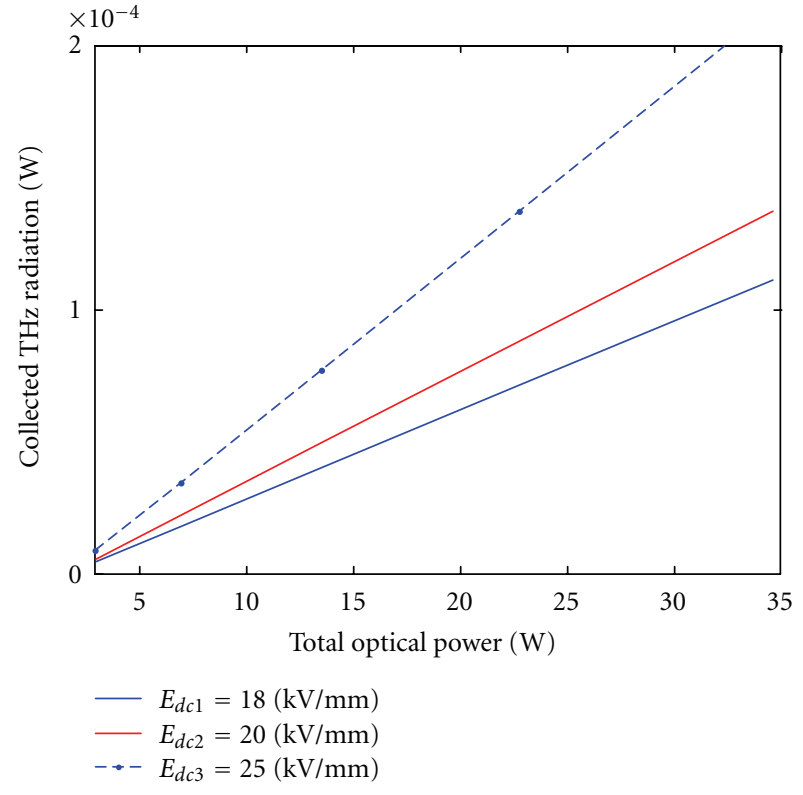

FIgURE 2: The power of the collected $\mathrm{THz}$ radiation utilizing a $5 \mathrm{~cm}$ diameter lens.

of the collected $\mathrm{THz}$ radiation. In the calculation, a lens of a diameter of $5 \mathrm{~cm}$ is assumed. Given these parameters, the collected power is $14.5 \%$ of the total generated power.

As can be seen from the numerical simulation, the higher the optical input power, the higher the generated $\mathrm{THz}$ radiation. This is because the fiber nonlinearity is relatively weak. We therefore proposed to utilize a ring resonator, incorporating a polarization coupler, to accumulate and boost the optical power. The optical power can hereby be effectively increased, yet using low power optical input sources. The proposed structure is depicted in Figure 3. In the structure, the polarization controllers at the outputs of the laser sources are adjusted such that the optical carriers are directed from $\operatorname{arm} a$ to $\operatorname{arm} b$. However, the polarization controller in the ring resonator is adjusted such that the optical carriers are directed from $\operatorname{arm} c$ to $\operatorname{arm} b$. Consequently, as both carriers lie within the FBG transmission band, the light power can be accumulated inside the ring resonator. To guarantee a constructive accumulation, a tunable time delay (TD) line is incorporated inside the ring resonator. Using the geometric series expression, one can get the optical power inside the ring resonator, given by

$$
\left|A^{b}\right|^{2}=\left|A^{a}\right|^{2} T \frac{1-\xi^{N}}{1-\xi} \times \frac{1-\xi^{* N}}{1-\xi^{*}},
$$

where $A^{a}$ and $A^{b}$ are the amplitudes of the two optical carriers at arm $a$ and $b, T$ is the transmission coefficient of the polarization coupler from arm $a$ to $\operatorname{arm} b, \xi=R L_{n} e^{j k Z}$, $R$ is the reflection coefficient from $\operatorname{arm} c$ to $\operatorname{arm} b, L_{n}$ is the total loss of the ring, $Z$ is the ring length, $k$ is the effective propagation constant, and $N$ is the number of the effective light rotation inside the resonator.

Let us assume $T=0.8, f_{1}=192.8 \mathrm{THz}$, and $Z=3 \mathrm{~m}$. The optical power ratio, defined as the ratio between the

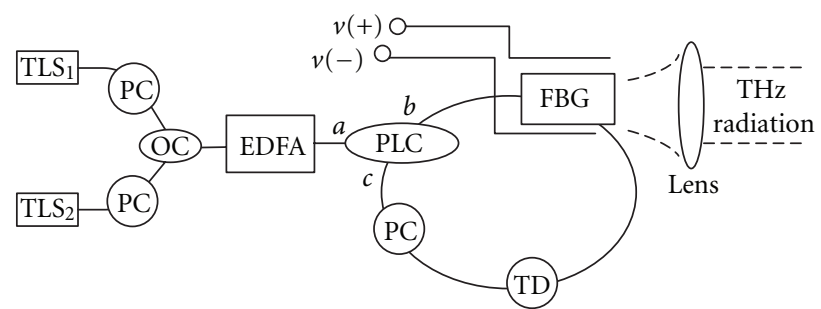

FIGURE 3: The structure of the proposed $\mathrm{THz}$ generation system. TLS: tunable laser source. OC: optical coupler, EDFA: erbiumdoped fiber amplifier, PLC: polarization coupler, FBG: fiber Bragg grating, TD: tunable time delay, PC: polarization controller.

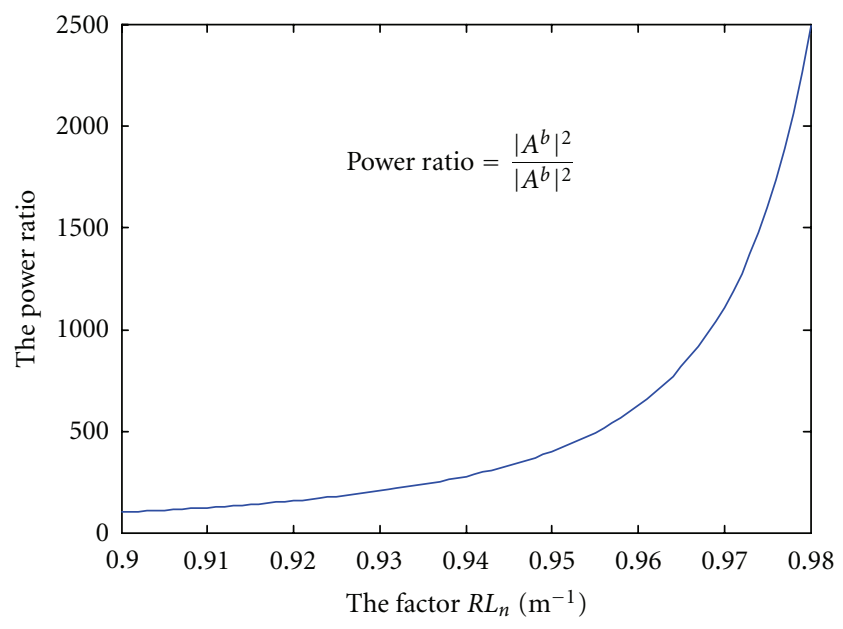

Figure 4: The power ratio between the power at $\operatorname{arm} b$ and $\operatorname{arm} a$ of the polarization coupler for the optical carrier $f_{1}$. Here, the power ratio is calculated as a function of the parameter $R L_{n}$.

optical power inside the ring resonator and the input optical power, is calculated, and is shown in Figure 4. Here the power ratio is calculated as a function of $R L_{n}$. As can be seen, the optical power can be boosted by maximizing this parameter, which implies a condition of having low losses inside the ring resonator and strong reflection at arm $c$ of the polarization coupler.

On the other hand, the power accumulation inside the resonator depends on the optical carrier frequency. The structure shown in Figure 3, however, employs two optical carriers. To optimize the total optical power inside the resonator and enhance the total power ratio for both optical carries, a tunable optical time delay (TD) is incorporated. Figure 5 shows the optical power ratio for both optical carriers, $f_{1}=192.8 \mathrm{THz}$ and $f_{2}=188.8 \mathrm{THz}$, versus the length change of the ring resonator. As can be seen from Figure 5, the total optical intensity inside the resonator (which is the sum of the intensities of the two optical carriers) can be boosted upon a relevant choice of the ring resonator length. This can be achieved via controlling the tunable optical time delay.

We note here that several other schemes can be implemented to enhance the power of the generated $\mathrm{THz}$ waves. First, an intensive pulsed laser input can be utilized as one of 


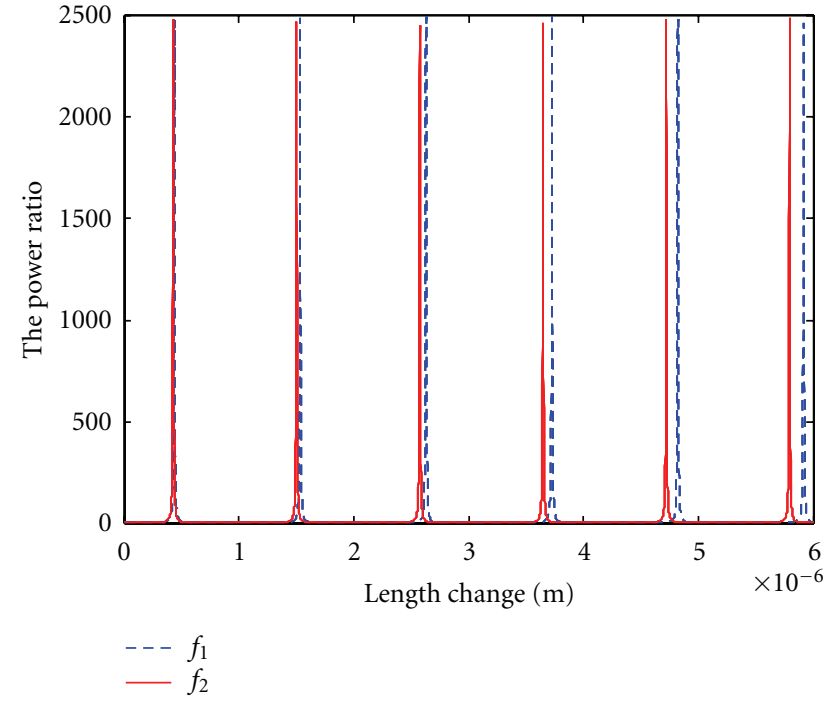

Figure 5: The power ratio for the two optical carriers $f_{1}$ and $f_{2}$ as a function of the ring resonator length.

the optical carries to boost the $\mathrm{THz}$ radiation, thanks to the weak two-photon absorption (TPA) and the instantaneous nonlinearity of the optical fiber. Second, a $\mathrm{THz}$ waveguide can also be utilized to enhance the $\mathrm{THz}$ focusing, leading to an improved efficiency in collecting the generated $\mathrm{THz}$ radiation.

Although an external electrostatic field has been considered above, this is not necessarily to be the case. For example, a poled optical fiber, with internal electrostatic field, could be utilized. We stress here that utilizing a poled fiber has a tremendous advantages. For instant, it is a solution to avoid biasing fluctuations; resulted in generating a highquality $\mathrm{THz}$ waves. Also, having an internal electrostatic field allows vital applications such as in-vivo $\mathrm{THz}$ scanning. Furthermore, avoiding an external circuitry enhances the system compatibility and portability.

The frequency of the generated $\mathrm{THz}$ radiation can be tuned by controlling the frequency spacing between the two optical carriers. However, as the system is designed to phase match the $\mathrm{THz}$ generation at given frequency (e.g., $4 \mathrm{THz}$ in the above simulation), the generation efficiency is expected to drop for any other $\mathrm{THz}$ frequencies. To mitigate this effect and to achieve a wide range of frequency-tunability, we choose to keep the optical carrier $\omega_{1}$ (which is close to the FBG central frequency) unchanged and tune the optical carrier $\omega_{2}$ (which lies far from the FBG central frequency). Figure 6 shows the numerical simulation of the normalized $\mathrm{THz}$ power versus the frequency of the generated $\mathrm{THz}$ wave. Here, same parameters (FBG specification, etc.) of the simulation presented in Figure 2 have been assumed. As can be seen the $\mathrm{THz}$ radiation can be generated over a large range from 1 to $30 \mathrm{THz}$. The maximum efficiency is achieved at $4 \mathrm{THz}$, while at $30 \mathrm{THz}$ the drop in efficiency is only $22 \%$. However, the efficiency is dramatically reduced for frequencies below $4 \mathrm{THz}$. This can be explained by noting that, for a frequency above $4 \mathrm{THz}$, the carrier at $\omega_{2}$ is shifted

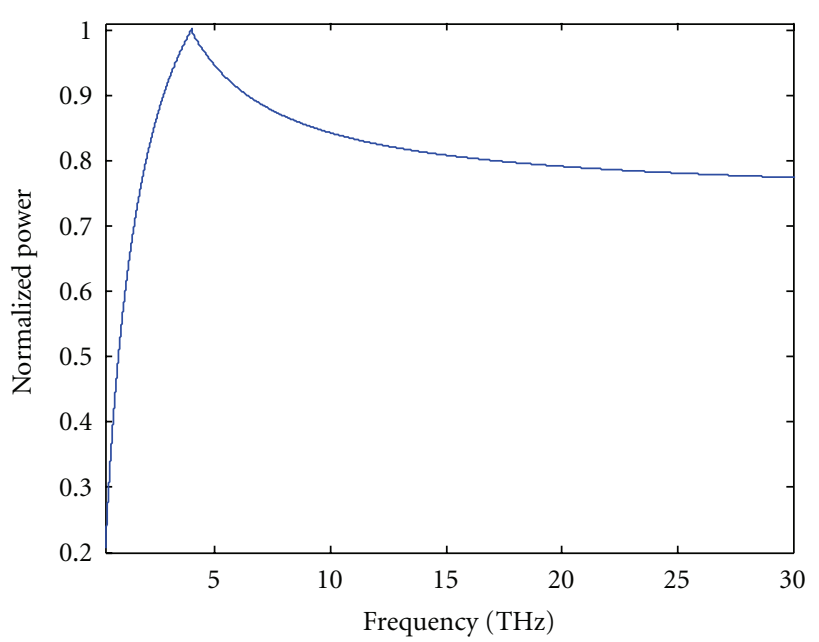

FIGURE 6: The normalized average THz power versus the frequency of the generated $\mathrm{THz}$ wave.

away from the FBG central frequency, thus the dispersion of the FBG will only have an impact on the carrier at $\omega_{1}$ and the phase matching condition is always satisfied. However, for a frequency below $4 \mathrm{THz}$, the optical carrier at $\omega_{2}$ is shifted towards the FBG central frequency, thus the dispersion of the FBG will have an impact on both the carriers at $\omega_{2}$ and $\omega_{1}$, and the phase matching condition is no longer satisfied.

\section{Conclusion}

We have proposed and theoretically investigated a novel approach to generating $\mathrm{THz}$ radiation in an optical fiber. An external electrostatic field is applied to the optical fiber to induce a second-order optical nonlinearity via the Kerr effect. Two optical carriers were introduced to the optical fiber and thus a $\mathrm{THz}$ radiation can be generated by the means of difference frequency generation. A FBG was utilized as a strong dispersive element to achieve the phase-match condition, to increase the generation efficiency. Furthermore, a ring resonator, incorporating a polarization coupler (PLC), was utilized to boost the optical power, which resulted in an increase in the $\mathrm{THz}$ power.

The generated $\mathrm{THz}$ wave could cover a wide frequency range utilizing a given FBG. The key on achieving this large tunable range (for a given FBG) is that one optical carrier was placed far away from the center frequency of the FBG. When the frequency of this optical carrier was tuned, the frequency of the generated $\mathrm{THz}$ wave was tuned, but the phase match condition was always preserved over the tunable range, assuring a relative constant $\mathrm{THz}$ power. We finally note that a poled optical fiber with internal electrostatic field can be used in lieu of the optical fiber with an external electrical bias. The system stability, compatibility, and portability can thus be enhanced. 


\section{References}

[1] G. P. Williams, "Filling the THz gap—high power sources and applications," Reports on Progress in Physics, vol. 69, no. 2, pp. 301-326, 2006.

[2] P. H. Siegel, "Terahertz technology in biology and medicine," IEEE Transactions on Microwave Theory and Techniques, vol. 52, no. 10, pp. 2438-2447, 2004.

[3] T. Nagatsuma, "Generating millimeter and terahertz waves," IEEE Microwave Magazine, vol. 10, no. 4, pp. 64-74, 2009.

[4] M. Tonouchi, "Cutting-edge terahertz technology," Nature Photonics, vol. 1, no. 2, pp. 97-105, 2007.

[5] B. S. Williams, "Terahertz quantum-cascade lasers," Nature Photonics, vol. 1, no. 9, pp. 517-525, 2007.

[6] A. Maestrini, J. S. Ward, J. J. Gill et al., "A frequency-multiplied source with more than $1 \mathrm{~mW}$ of power across the 840900-GHz band," IEEE Transactions on Microwave Theory and Techniques, vol. 58, no. 7, pp. 1925-1932, 2010.

[7] A. Dobroiu, M. Yamashita, Y. N. Ohshima, Y. Morita, C. Otani, and K. Kawase, "Terahertz imaging system based on a backward-wave oscillator," Applied Optics, vol. 43, no. 30, pp. 5637-5646, 2004.

[8] D. R. Chamberlin, E. Brundermann, and E. E. Haller, "Narrow linewidthintervalence-band emission from germanium terahertz lasers," Applied Physics Letters, vol. 83, no. 3, pp. 3-5, 2003.

[9] P.-C. Lv, R. T. Troeger, S. Kim et al., "Terahertz emission from electrically pumped gallium doped silicon devices," Applied Physics Letters, vol. 85, no. 17, pp. 3660-3662, 2004.

[10] N. N. Zinov'ev, A. S. Nikoghosyan, R. A. Dudley, and J. M. Chamberlain, "Conversion of short optical pulses to terahertz radiation in a nonlinear medium: experiment and theory," Physical Review B, vol. 76, no. 23, Article ID 235114, 2007.

[11] L. Miao, D. Zuo, Z. Jiu, and Z. Cheng, "An efficient cavity for optically pumped terahertz lasers," Optics Communications, vol. 283, no. 16, pp. 3171-3175, 2010.

[12] B. A. Knyazev, G. N. Kulipanov, and N. A. Vinokurov, "Novosibirsk terahertz free electron laser: instrumentation development and experimental achievements," Measurement Science and Technology, vol. 21, no. 5, Article ID 054017, 2010.

[13] A. Rice, Y. Jin, X. F. Ma et al., "A Terahertz optical rectification from ¡110> zinc-blende crystals," Applied Physics Letters, vol. 64, no. 11, pp. 1324-1326, 1994.

[14] Y. Jiang, D. Li, Y. J. Ding, and I. B. Zotova, "Terahertz generation based on parametric conversion: from saturation of conversion efficiency to back conversion," Optics Letters, vol. 36, no. 9, pp. 1608-1610, 2011.

[15] D. Dietze, J. Darmo, S. Roither, A. Pugzlys, J. N. Heyman, and K. Unterrainer, "Polarization of terahertz radiation from laser generated plasma filaments," Journal of the Optical Society of America B, vol. 26, no. 11, pp. 2016-2027, 2009.

[16] K. Saito, T. Tanabe, Y. Oyama, K. Suto, and J.-I. Nishizawa, "Terahertz-wave generation by $\mathrm{GaP}$ rib waveguides via collinear phase-matched difference-frequency mixing of nearinfrared lasers," Journal of Applied Physics, vol. 105, no. 6, Article ID 063102, 2009.

[17] Y. Sasaki, A. Yuri, K. Kawase, and H. Ito, "Terahertz-wave surface-emitted difference frequency generation in slantstripe-type periodically poled $\mathrm{LiNbO}_{3}$ crystal," Applied Physics Letters, vol. 81, no. 18, pp. 3323-3325, 2002.

[18] M. Yi, K. Lee, J. Lim, Y. Hong, Y.-D. Jho, and J. Ahn, “Terahertz waves emitted from an optical fiber," Optics Express, vol. 18, no. 13, pp. 13693-13699, 2010.
[19] M. Qasymeh, M. Cada, and S. A. Ponomarenko, "Quadratic electro-optic Kerr effect: applications to photonic devices," IEEE Journal of Quantum Electronics, vol. 44, no. 8, pp. 740746, 2008.

[20] H. An and S. Fleming, "Inverstigating the effectiveness of thermally poling optical fibers with various internal electrode configurations," Optics Express, vol. 20, no. 7, pp. 7436-7444, 2012.

[21] G. P. Agrawal, Nonlinear Fiber Optics, Academic Press, San Diego, Calif, USA, 4th edition, 2007.

[22] S. Pereira and J. E. Sipe, "Nonlinear pulse propagation in birefringent fiber Bragg gratings," Optics Express, vol. 3, no. 11, pp. 418-432, 1998.

[23] Y. S. Kivshar and G. P. Agrawal, Optical Solitons: from Fibers to Photonic Crystals, Academic Press, San Diego, Calif, USA, 2003.

[24] E. A. Saleh and M. C. Teich, Fundamental of Photonics, John Wiley \& Sons, New York, NY, USA, 1991. 

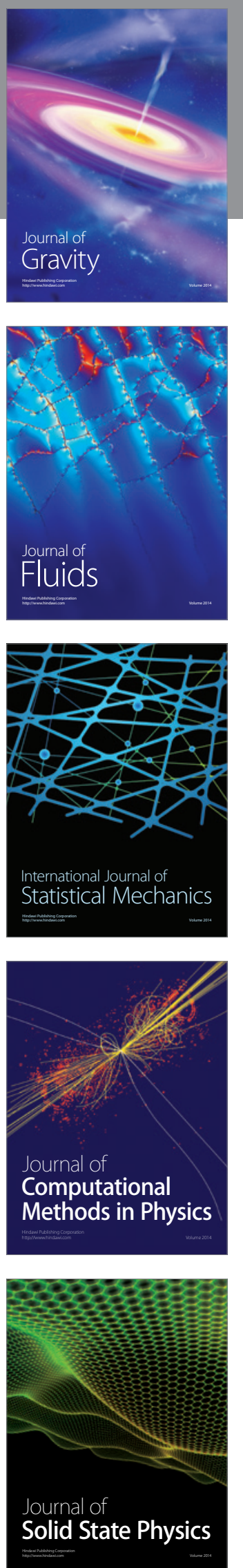

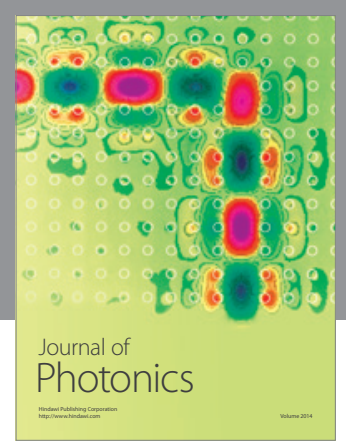

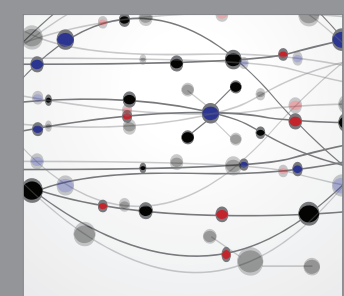

The Scientific World Journal
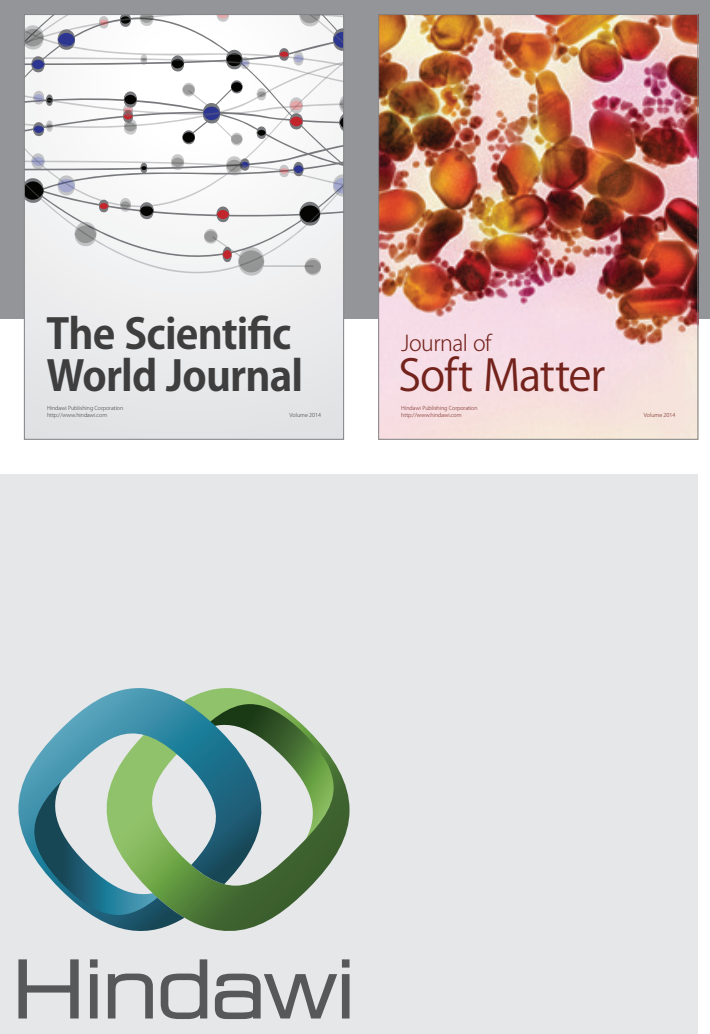

Submit your manuscripts at

http://www.hindawi.com
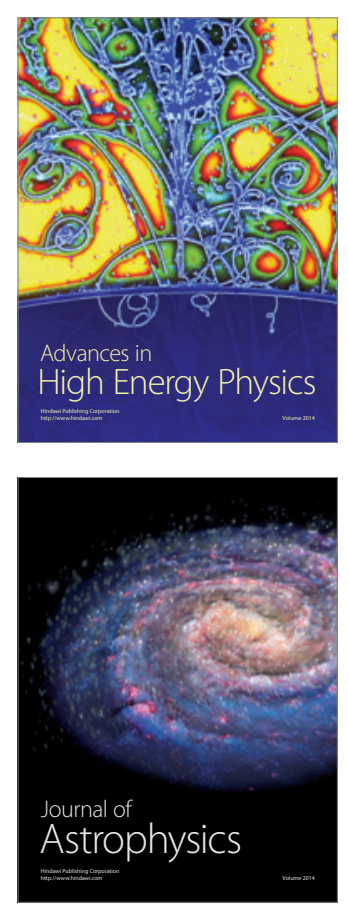
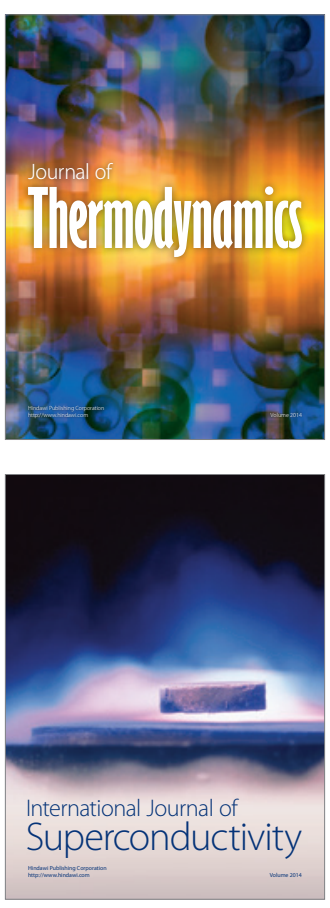
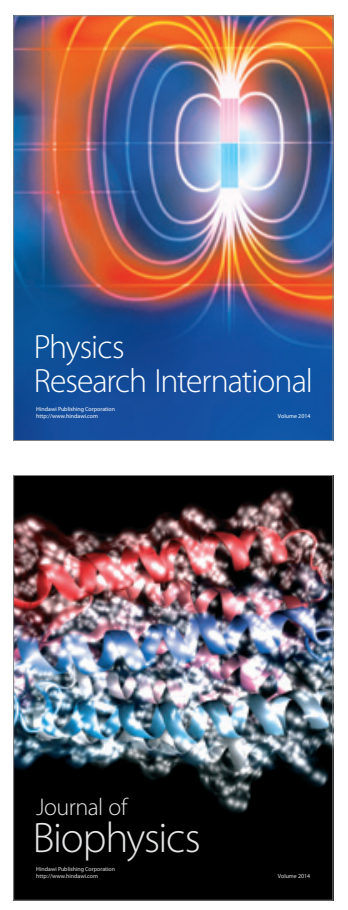
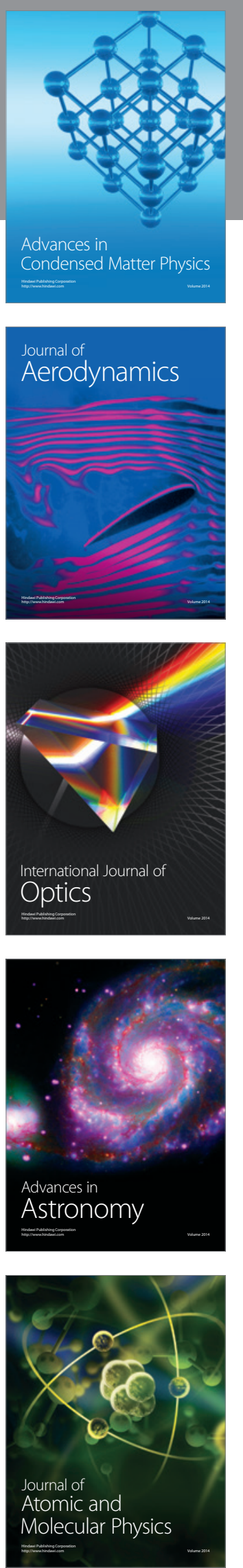